\title{
Causas y consecuencias de la insatisfacción en consumidores con atribuciones externas ${ }^{1}$
}

\author{
Causes and consequences of consumer \\ dissatisfaction with external attributions
}

\author{
Beatriz Moliner Velázquez ${ }^{2}$ \\ María Fuentes Blasco 3 \\ Universidad de Valencia / Universidad Pablo de Olavide
}

Recibido el 1 de julio de 2009, aceptado el 20 de octubre de 2010

$\mathrm{N}^{\circ}$ de clasificación JEL: M1, M3

DOI: $10.5295 / \mathrm{cdg} .100165 \mathrm{bm}$

Reseña bibliográfica: MOLINER, B. y FUENTES, M. (2011): "Causas y consecuencias de la insatisfacción en consumidores con atribuciones externas", Cuadernos de Gestión, Vol 11, nº 1, pp.37-58, DOI: $10.5295 / \mathrm{cdg} .100165 \mathrm{bm}$

\section{Resumen:}

Este trabajo aborda el estudio de la formación de la insatisfacción, desde la perspectiva cognitiva y afectiva, $y$ de sus consecuencias en forma de intenciones de comportamientos de queja. El objetivo es investigar la influencia directa e indirecta, a través de los afectos negativos, que ejerce la desconfirmación de expectativas sobre la insatisfacción, y analizar la contribución de estos juicios sobre las intenciones de respuestas de queja, a terceras partes y privadas. A partir de una muestra de clientes insatisfechos con restaurantes que manifiestan distintos niveles de atribución externa, se ha construido un modelo causal para estudiar las relaciones. Los resultados confirman la existencia de un efecto directo de la desconfirmación sobre la insatisfacción y sobre los afectos, y un efecto directo de dicha insatisfacción sobre las intenciones posteriores, no existiendo diferencias significativas según el grado de atribución externa. Las conclusiones sugieren implicaciones para la gestión y futuras líneas de investigación.

Palabras clave:

Insatisfacción, desconfirmación, afectos y comportamiento de queja.

\begin{abstract}
:
This paper deals with the process of how consumer dissatisfaction is formed, both from a cognitive and an affective perspective, and also with how it translates into complaint behavior intentions. Our main objective is, on

\footnotetext{
${ }^{1}$ Este estudio ha sido realizado con el apoyo financiero prestado por el proyecto I+D del Plan Nacional SEJ200766054/ECON.

${ }^{2}$ Universidad de Valencia. Departamento de Comercialización e Investigación de Mercados, Facultad de Economía. Av. Dels Tarongers, s/n, Edificio Departamental Oriental, 46022 Valencia. Correo electrónico: beatriz.moliner@uv.es

${ }^{3}$ Universidad Pablo de Olavide. Departamento de Dirección de Empresas, Facultad de Ciencias Empresariales. Ctra. de Utrera, km. 1, 41013 Sevilla. Correo electrónico: mfuebla@upo.es
} 
Causas y consecuencias de la insatisfacción en consumidores con atribuciones externas

the one hand, to gain an insight into the effect that the disconfirmation of expectations has on dissatisfaction judgments, not only directly, but also indirectly through negative affects. On the other hand, we intend to analyze the influence these judgments exert on complaint behavior intentions, which can develop into complaint responses, third party responses, or private responses. From a sample of customers who have experienced an unsatisfactory situation with a restaurant and show different external attribution level, we have put forward a causal model to study the causal relationships. The results confirm a direct effect of disconfirmation on dissatisfaction and on affects, a direct effect of that dissatisfaction on complaint behaviour intentions and there are not significant differences according to external attribution level. The findings suggest management implications and future research.

\section{Key Words:}

Dissatisfaction, disconfirmation, affects and complaining behaviour. 


\section{INTRODUCCIÓN}

El comienzo del estudio de la satisfacción se puede situar a finales de la década de los sesenta siendo en los años ochenta cuando se produce la verdadera eclosión de investigaciones. A pesar de verse frenado este auge en la siguiente década, actualmente continúa teniendo un interés notable (Ekinci et al., 2008). La literatura sobre satisfacción aborda el estudio de los fenómenos relacionados con los juicios que realizan los consumidores, tanto en sentido positivo (satisfacción) como en sentido negativo (insatisfacción). No obstante, el cuerpo teórico muestra cierta tendencia a la investigación sobre las valoraciones positivas.

El análisis de los trabajos más relevantes indica que no existe una diferencia clara entre el estudio de la satisfacción y de la insatisfacción ya que los autores suelen aportar enfoques, bases teóricas y resultados empíricos que, en principio, parecen ser útiles para explicar ambos juicios. Giese y Cote (2000; p. 19) afirman que la revisión de la literatura muestra una menor preocupación por la conceptualización de la insatisfacción del consumidor, señalando que la razón radica en el enfoque unidimensional de la satisfacción compartido por la mayoría de los investigadores. Según este enfoque, este fenómeno es una variable bipolar compuesta por diferentes grados ubicados dentro de un continuo y delimitado por dos extremos opuestos: la satisfacción y la insatisfacción. Este planteamiento implica que ambas dimensiones pueden ser definidas de forma similar y formarse a través de idénticos mecanismos (Mittal et al., 1999; Jun et al., 2001).

Sin embargo, algunas aportaciones sugieren que se trata de fenómenos que difieren en algunas cuestiones conceptuales y operativas (Giese y Cote, 2000). Trabajos de diferentes disciplinas han señalado que la presencia de algunos factores aumenta la satisfacción pero su ausencia no aumenta la insatisfacción y que la ausencia de algunos factores aumenta la insatisfacción pero su presencia no aumenta la satisfacción (Vargo et al., 2007). En este sentido, existen indicios de que la insatisfacción puede ser una respuesta formada a partir de un conjunto de variables que no tienen porque coincidir siempre con los antecedentes de la satisfacción. De hecho, algunos autores señalan que ambos fenómenos deben ser entendidos y medidos como dimensiones bipolares distintas (Prakash, 1991; Westbrook y Oliver, 1991; Mano y Oliver, 1993; Theng y Ng, 2001). Investigaciones recientes encuentran una relación asimétrica entre la satisfacción e insatisfacción (James, 2007) e incluso ausencia de relación entre ambas variables (Vargo et al., 2007). También la diferencia existente entre las consecuencias de la satisfacción y la insatisfacción son un argumento más para defender el enfoque multidimensional del constructo satisfacción. En esta línea, las respuestas a la insatisfacción, denominadas también "comportamiento de queja" (Singh, 1990), constituyen un área de especial interés en Marketing por sus implicaciones en campos muy diversos.

Respecto a este debate sobre el carácter dimensional de la satisfacción, nos sumamos al enfoque que diferencia ambos fenómenos como dimensiones distintas y consideramos que la insatisfacción merece un análisis independiente para examinar su origen y sus consecuencias. Es por ello que nuestro trabajo pretende ser una aportación al estudio de la insatisfacción considerando que ciertas cuestiones teóricas y operativas difieren del estudio de la satisfacción. Frente al enfoque bipolar insatisfacción-satisfacción, nuestro planteamiento implica una mejor adaptación conceptual y operativa de la insatisfacción al contexto de 
análisis. Conceptualmente, y desde la perspectiva cognitiva, el proceso de desconfirmación de expectativas es la teoría que mayor interés ha tenido en la literatura (Oliver, 1980). Sin embargo, las atribuciones causales también ha sido un enfoque utilizado con frecuencia para explicar la satisfacción e insatisfacción (Casado et al., 2004; Tsiros et al., 2004) y, especialmente, las atribuciones de origen externo se han considerado como las valoraciones que mayor relación guardan con los niveles de insatisfacción (Oliver y DeSarbo, 1988). También, desde la perspectiva de las emociones y, sobre todo, en el ámbito de los servicios, el componente afectivo ha permitido completar el proceso de formación utilizando los afectos como variable determinante (Bagozzi et al., 1999; Bougie et al., 2003; Bigné y Andreu, 2004). A nivel operativo, ha sido frecuente a la vez que criticado el uso de escalas simples para medir el grado de insatisfacción-satisfacción (Mittal et al., 1999; Kattara et al., 2008), sin embargo, el empleo de medidas más adaptadas para medir la insatisfacción permite recoger mejor la naturaleza de este concepto.

De acuerdo con este planteamiento, nuestro trabajo consiste en el estudio de las causas y consecuencias de la insatisfacción en sujetos con experiencias insatisfactorias en restaurantes que realizan atribuciones externas. Concretamente, por una parte, pretendemos analizar el componente cognitivo y afectivo de los juicios de insatisfacción investigando el efecto directo e indirecto, a través de los afectos, que tiene la desconfirmación de expectativas. De esta forma, se conseguirá conocer la contribución que tiene el proceso cognitivo de la evaluación de la experiencia en la formación de los afectos y, a su vez, en la explicación de los juicios de insatisfacción. Y por otra parte, analizamos la contribución que tiene dicha insatisfacción sobre las intenciones de comportamientos de queja manifestados a través de respuestas de queja (al proveedor), a terceras partes (a organismos de protección del consumidor) y privadas (conducta de cambio y comentarios boca-oreja negativos). Por último, también pretendemos conocer diferencias en estas relaciones en función del nivel de atribución externa del consumidor para comprobar el efecto moderador de esta variable en la formación y las consecuencias de la insatisfacción.

\section{MARCO TEÓRICO Y FORMULACIÓN DE HIPÓTESIS}

\subsection{Componente cognitivo y afectivo de la insatisfacción}

La literatura ha empleado diferentes enfoques para identificar los antecedentes de los juicios de satisfacción e insatisfacción. Tradicionalmente, el proceso de formación se ha explicado a través de procesos cognitivos basados en el proceso de desconfirmación (Oliver, 1980). Diversas definiciones de satisfacción recogen este componente indicando que se trata de una respuesta evaluativa a una experiencia de compra o consumo (Jonson et al., 1995). Respecto al proceso de evaluación, la literatura recoge diferentes estándares de comparación con la performance del producto siendo las expectativas predictivas la variable predominante (Cadotte et al., 1987; Spreng et al., 1996).

El paradigma de desconfirmación de expectativas supone un punto de referencia en el desarrollo de numerosas investigaciones que explican el origen de la satisfacción e insatis- 
facción a partir de la intensidad y dirección de la diferencia entre las expectativas previas y la performance del producto (Erevelles y Leavitt, 1992; Woodruff et al., 1993; Oliver y DeSarbo, 1998; Oliver et al., 1997). Múltiples aportaciones empíricas han demostrado el efecto directo de la desconfirmación de expectativas sobre los niveles de satisfacción (Anderson y Sullivan, 1993; Andreassen, 2000; Bowen, 2001; Bigné y Andreu, 2002). Trabajos más recientes en el ámbito de los servicios también han encontrado una influencia relevante de la desconfirmación de expectativas sobre los juicios de satisfacción e insatisfacción (Roch y Poister 2006; James, 2007). Considerando este planteamiento en el contexto de experiencias insatisfactorias, formulamos la primera hipótesis:

$H_{1}$ : La desconfirmación negativa de expectativas influye positivamente en el nivel de insatisfacción.

Progresivamente la literatura ha ido sugiriendo un doble componente cognitivo-afectivo de la satisfacción. En este sentido, a partir de los ochenta las investigaciones comienzan a destacar que las variables afectivas pueden tener también una fuerte influencia (Westbrook, 1987). Ello se ve reflejado en algunas conceptuaciones de satisfacción que definen este constructo como una respuesta emocional o un sentimiento que se forma de los resultados de una experiencia de compra o consumo (Woodruff y Gardial, 1996; Giese y Cote, 2000). También diferentes estudios han confirmado la importancia que tienen los sentimientos derivados de una experiencia en la formación de la satisfacción e insatisfacción y en las respuestas posteriores (Oliver et al., 1997; Liljander y Strandvik, 1997). Investigaciones más recientes han permitido reforzar este carácter afectivo de la satisfacción complementando así el enfoque cognitivo tradicional (Chebat y Michon, 2003; Bigné y Andreu, 2004).

Algunas investigaciones muestran que, en determinadas situaciones, las respuestas afectivas que resultan de una experiencia influyen más en los juicios de satisfacción e insatisfacción que la desconfirmación de expectativas (Dubé-Rioux, 1990; Jun et al., 2001). Además, la debilidad explicativa de la valoración cognitiva destaca especialmente en los servicios, cobrando mayor fuerza el rol de las emociones, debido a la elevada interacción del usuario con los elementos del servicio y con el personal. Es por ello que este enfoque haya tenido mayor atención en el área de la insatisfacción, el comportamiento de queja y la satisfacción con el tratamiento de las quejas (Wirtz y Bateson, 1999; Maute y Dubé, 1999; Yi y Baumgartner, 2004).

Los afectos representan un conjunto de procesos mentales que generan emociones, estados de humor (Andreu, 2003) y, posiblemente, actitudes (Bagozzi et al., 1999). Se suelen considerar en la literatura como variables unidimensionales bipolares, ya que cada uno de ellos representa un tipo de sentimiento, bien de carácter positivo, negativo o neutral, por lo que pueden coexistir afectos de diferente naturaleza (Oliver, 1993). En general, las investigaciones indican que la intensidad de los afectos que aparecen en una experiencia de compra tiene una relación positiva con el nivel de satisfacción (Wirtz et al., 2000; Mattila y Ro, 2008). Por tanto, proponemos la siguiente hipótesis:

\section{$\mathrm{H}_{2}$ : Los afectos negativos influyen positivamente en el nivel de insatisfacción.}

Algunas investigaciones sobre emociones consideran que el tipo y la intensidad de los afectos que genera una experiencia de compra se combinan con la desconfirmación de expectativas (Wesbrook, 1987; Oliver, 1993). De hecho, se pueden encontrar definicio- 
nes de satisfacción que indican que la respuesta afectiva que provoca una experiencia de compra o consumo es el resultado de la evaluación o comparación entre la performance y un determinado estándar (Cadotte et al., 1987; Spreng et al., 1996). También en algunas investigaciones empíricas se pone de manifiesto la relación positiva que existe entre los afectos y la desconfirmación de expectativas (Oliver et al., 1997; Wirtz y Bateson, 1999; Menon y Dubé, 2000; Bigné y Andreu, 2002). Estos trabajos demuestran que la intensidad de la desconfirmación tiene un efecto positivo sobre la intensidad de los afectos. Del mismo modo, en la literatura sobre las teorías de las emociones del consumidor, existen autores que destacan la evaluación como un elemento cognitivo que permite explicar parte de la naturaleza de la emoción (Ortony et al., 1988; Lazarus, 1991). Trasladando estos planteamientos al contexto de la insatisfacción, entendemos que la desconfirmación negativa de expectativas que provoca una experiencia insatisfactoria genera una serie de afectos negativos. Por tanto, la desconfirmación ejercería una influencia no sólo directa sobre la insatisfacción, sino también indirecta a través de los afectos negativos. La hipótesis que formulamos al respecto es:

$\mathrm{H}_{3}$ : La desconfirmación negativa de expectativas influye positivamente en los afectos negativos que tienen los sujetos.

\subsection{La insatisfacción y las intenciones de comportamientos de queja}

Los modelos clásicos sobre la formación de la satisfacción ya ponían de manifiesto el efecto de estos juicios sobre las actitudes e intenciones de los consumidores. Posteriormente, numerosas investigaciones se han apoyado en estos planteamientos para estudiar las intenciones conductuales después de una experiencia de compra (Anderson y Sullivan, 1993). En el área de los servicios, ha habido un interés especial por comprender las relaciones entre la satisfacción y las intenciones futuras (Choi et al., 2002; Bigné y Andreu, 2002) y los comportamientos (Onyeaso, 2007).

En el contexto de la insatisfacción y el comportamiento de queja, las investigaciones se centran principalmente en el estudio de las variables que influyen en la elección de las respuestas a la insatisfacción, generalmente diferenciadas en respuestas privadas y públicas (de queja y a terceras partes) (Crié, 2003). Sin embargo, también se pueden encontrar aportaciones que analizan la contribución de la insatisfacción sobre las intenciones de desarrollar una repetición de compra o una conducta de queja (Mittal et al., 1999; Mittal y Kamakura, 2001; Oh, 2006). En general, estos trabajos comprueban que el nivel de insatisfacción tiene una relación positiva con las intenciones que muestran los consumidores hacia la conducta de cambio, los comentarios boca-oreja negativos y la manifestación de la queja, ya sea al proveedor del producto o servicio como a terceras partes. Por tanto, planteamos la siguiente hipótesis:

$\mathrm{H}_{4}$ : El nivel de insatisfacción influye positivamente en las intenciones de comportamientos de queja.

En síntesis, las hipótesis relativas a las relaciones causales que se pretenden analizar en situaciones de elevada atribución externa se muestran en la figura 1. 
Figura 1

Modelo propuesto sobre las causas y consecuencias de la insatisfacción

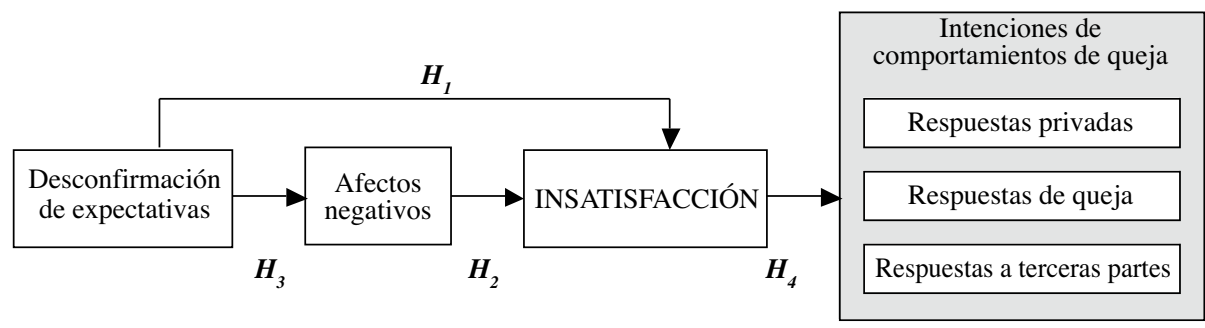

\section{METODOLOGÍA DE INVESTIGACIÓN}

De acuerdo con los objetivos planteados, desarrollamos una investigación de carácter causal, ya que se aborda el estudio de los antecedentes y las consecuencias de la insatisfacción analizando las relaciones causales de la secuencia de variables que se presenta en la figura 1. En la tabla 1 se sintetizan las principales características del estudio.

El contexto que utilizamos en esta investigación es el de los restaurantes. Se ha considerado un sector de gran interés por su capacidad de generar fácilmente experiencias insatisfactorias en función del nivel de implicación del individuo, así como evaluaciones críticas de los resultados del servicio. Además, se trata de un sector amplio en cuanto a número de usuarios e incidentes que provocan niveles diferentes de placer y estimulación en los individuos. Este ámbito de aplicación se ha utilizado con frecuencia en la literatura sobre insatisfacción (Morel et al., 1997; Wirtz et al., 2000; Liu y McClure, 2001; Mattila y Ro, 2007).

Tabla 1

Ficha técnica de la investigación

\begin{tabular}{ll}
\hline Enfoque de la investigación & Exploratorio \\
Método de obtención de información & Encuesta ad-hoc \\
Método de contacto & $\begin{array}{l}\text { Encuesta auto-administrada } \\
\text { Sujetos que han experimentado una insatisfacción con un } \\
\text { Universo }\end{array}$ \\
restaurante \\
Población objetivo & $\begin{array}{l}\text { Individuos de una ciudad española de edad entre 20 y } 64 \\
\text { años }\end{array}$ \\
Método de muestreo & Muestreo por conveniencia \\
Tamaño de la muestra & 380 sujetos \\
Error muestral aceptable & $\pm 5 \%$ \\
Fiabilidad & $95,5 \%$ \\
Programa estadístico & LISREL 8.51 \\
Técnicas estadísticas & Análisis causal \\
\hline
\end{tabular}


Se ha recurrido al método del incidente crítico (Roos, 2002), por lo que los sujetos objeto de estudio han tenido que recordar con claridad una situación insatisfactoria específica que ha servido de punto de referencia para la valoración de las variables que se analizan. Diversas investigaciones en este ámbito han recurrido a esta técnica (Folkes, 1984; Maxham III y Netemeyer, 2002; Zeelenberg y Pieters, 2004).

El método de investigación aplicado tiene carácter cuantitativo y está basado en una encuesta ad-hoc. El cuestionario empleado es estructurado y ha permitido recoger la información de forma auto-administrada. Dicho cuestionario se compone de diferentes escalas utilizadas en diversas investigaciones sobre insatisfacción y adaptadas a nuestro contexto de análisis (Tabla 2). Para medir la desconfirmación de expectativas, se empleó una escala simple de cinco puntuaciones que permitía recoger la comparación entre los resultados obtenidos y los esperados (Oliver, 1981; Babin et al., 1994; Esteban et al., 2002). Y en el resto de variables, se recurrió a escalas de tipo Likert con cinco puntuaciones para recoger la intensidad de la insatisfacción, los afectos negativos, las intenciones de comportamientos de queja y las atribuciones externas.

La población de referencia para la selección de la muestra está formada por individuos de una ciudad española de edad comprendida entre 20 y 64 años. Se ha realizado un muestreo de conveniencia, recogiendo 380 cuestionarios válidos.

Tabla 2

Escalas de medida empleadas en el cuestionario

\begin{tabular}{|l|l|l|}
\cline { 2 - 3 } \multicolumn{1}{c|}{} & \multicolumn{1}{|c|}{ Items de las escalas } & \multicolumn{1}{|c|}{ Fuentes } \\
\hline \multirow{2}{*}{ Insatisfacción } & $\begin{array}{l}\text { Insatisfacción 1: Este es uno de los peores restaurantes que } \\
\text { he visitado } \\
\text { Insatisfacción 2: No estoy satisfecho de haber visitado este } \\
\text { restaurante } \\
\text { Insatisfacción 3: No ha sido acertado acudir a este restau- } \\
\text { rante } \\
\text { Insatisfacción 4: No he disfrutado en este restaurante } \\
\text { Insatisfacción 5: Me arrepiento de haber estado en ese } \\
\text { restaurante }\end{array}$ & $\begin{array}{l}\text { Adaptada } \\
\text { de Bigné y } \\
\text { Andreu (2002; } \\
\text { p. 502) }\end{array}$ \\
\hline $\begin{array}{l}\text { Desconfirmación } \\
\text { de expectativas }\end{array}$ & $\begin{array}{l}\text { En relación con sus expectativas, su experiencia en el } \\
\text { restaurante fue ... de lo que esperaba: 1-Justo, 2-Algo peor, } \\
\text { 3-Peor, 4- Bastante peor, 5- Mucho peor }\end{array}$ & $\begin{array}{l}\text { Adaptada de } \\
\text { Oliver (1981; } \\
\text { p. 40) }\end{array}$ \\
\hline Afectos negativos & $\begin{array}{l}\text { Afecto 1: Enfadado } \\
\text { Afecto 2: Despreciado } \\
\text { Afecto 3: Furioso } \\
\text { Afecto 4: Indignado } \\
\text { Afecto 5: Menospreciado }\end{array}$ & $\begin{array}{l}\text { Tomada par- } \\
\text { cialmente de } \\
\text { Izard (1991) } \\
\text { basada en la } \\
\text { escala DES II } \\
\text { (Izard, 1977) }\end{array}$ \\
\hline
\end{tabular}




\begin{tabular}{|l|l|l|}
\cline { 2 - 3 } \multicolumn{1}{c|}{} & \multicolumn{1}{|c|}{ Items de las escalas } & \multicolumn{1}{c|}{ Fuentes } \\
\hline & $\begin{array}{l}\text { Si volviera a ocurrir la misma situación en ese restaurante: } \\
\text { Intención 1: No volvería a visitar ese restaurante otra vez } \\
\text { Intención 2: Contaría a mis amigos y/o familiares mi mala } \\
\text { experiencia }\end{array}$ & \\
$\begin{array}{l}\text { Intenciones de } \\
\text { comportamientos } \\
\text { de queja }\end{array}$ & $\begin{array}{l}\text { Intención 3: Iría a otros restaurantes a partir de entonces } \\
\text { que no lo visiten } \\
\text { Intención 5: Discutiría el problema con el responsable del } \\
\text { restaurante }\end{array}$ & $\begin{array}{l}\text { Liua y McClure } \\
\text { (2001; p. 72) }\end{array}$ \\
\hline $\begin{array}{l}\text { Intención 6: Pediría que me solucionaran el problema } \\
\text { Intención 7: Informaría de mi problema a un organismo de } \\
\text { protección del consumidor }\end{array}$ & \\
\hline Atribuciones & $\begin{array}{l}\text { Atribución 1: El restaurante fue completamente responsa- } \\
\text { externas del problema que tuve } \\
\text { Atribución 2: En general, culpo al restaurante de ese } \\
\text { problema }\end{array}$ & $\begin{array}{l}\text { Adaptada de } \\
\text { Maxham III } \\
\text { y Netemeyer } \\
\text { (2002; p. 69) }\end{array}$ \\
\hline
\end{tabular}

La teoría de las atribuciones ha sido un enfoque ampliamente contrastado en multitud de investigaciones sobre insatisfacción desde hace más de 30 años (Stephens y Gwinner, 1998; Swanson y Kelley, 2001; Tsiros et al., 2004; Mattila y Ro, 2007). El individuo que busca las razones del fracaso de una compra realiza inferencias causales utilizando tres dimensiones: el origen (interno, externo y situacional), la estabilidad y el control (Folkes, 1984; Weiner, 1985, 2000; Oliver y DeSarbo, 1988). En general, las aportaciones que utilizan este enfoque coinciden en que las atribuciones de origen externo son las valoraciones que tienen más relación con los niveles de insatisfacción (Mittal y Kamakura, 2001; Oh, 2006).

Según este planteamiento, y con el objetivo de conocer el efecto moderador de esta variable, segmentamos la muestra en función de su nivel medio de atribución externa. Para operar de esta forma, creamos un índice medio de las variables que componen dicha dimensión (las variables atribución 1 y atribución 2 cargan en una sola dimensión, con índice de fiabilidad $\alpha=0,655)$, usando su valor medio muestral $(4,27)$ como punto de corte. Esto nos permitió segmentar la muestra de 380 consumidores en dos grupos: uno formado por 194 individuos que manifiestan elevada atribución externa y otro formado por 186 individuos con nivel bajo de atribuciones externas (Tabla 3).

Tabla 3

Valores medios y dispersión e las variables

\begin{tabular}{|l|c|c|c|c|c|c|c|}
\cline { 2 - 7 } \multicolumn{1}{c|}{} & \multicolumn{2}{c|}{$\begin{array}{c}\text { Total } \\
\mathrm{N}=380\end{array}$} & \multicolumn{2}{c|}{$\begin{array}{c}\text { Alto nivel de } \\
\text { atribuciones } \\
\text { (N=194) }\end{array}$} & \multicolumn{2}{c|}{$\begin{array}{c}\text { Bajo nivel de } \\
\text { atribuciones } \\
\text { (N=186) }\end{array}$} & \multicolumn{1}{c}{} \\
\cline { 2 - 8 } & Media & D.T. & Media & D.T. & Media & D.T. & F \\
\hline Insatisfacción 1 & 3,26 & 1,18 & 3,53 & 1,19 & 2,99 & 1,09 & $20,74^{* *}$ \\
\hline Insatisfacción 2 & 3,92 & 0,97 & 4,17 & 0,97 & 3,65 & 0,91 & $28,66^{* *}$ \\
\hline
\end{tabular}




\begin{tabular}{|c|c|c|c|c|c|c|c|}
\hline & \multicolumn{2}{|c|}{$\begin{array}{c}\text { Total } \\
\mathrm{N}=380\end{array}$} & \multicolumn{2}{|c|}{$\begin{array}{l}\text { Alto nivel de } \\
\text { atribuciones } \\
(\mathrm{N}=194)\end{array}$} & \multicolumn{2}{|c|}{$\begin{array}{l}\text { Bajo nivel de } \\
\text { atribuciones } \\
(\mathrm{N}=186)\end{array}$} & \multirow[b]{2}{*}{$\mathrm{F}$} \\
\hline & Media & D.T. & Media & D.T. & Media & D.T. & \\
\hline Insatisfacción 3 & 3,99 & 0,86 & 4,21 & 0,81 & 3,75 & 0,84 & $29,17^{* *}$ \\
\hline Insatisfacción 4 & 3,99 & 0,89 & 4,15 & 0,95 & 3,84 & 0,80 & $11,80^{* *}$ \\
\hline Insatisfacción 5 & 3,69 & 1,04 & 3,96 & 1,03 & 3,42 & 0,98 & $27,45^{* *}$ \\
\hline Desconfirmación & 3,50 & 1,05 & 3,88 & 0,92 & 3,11 & 1,04 & $57,13^{* *}$ \\
\hline Afecto 1 & 3,76 & 1,03 & 4,05 & 0,98 & 3,46 & 1,01 & $33,29^{* *}$ \\
\hline Afecto 2 & 3,04 & 1,19 & 3,34 & 1,20 & 2,72 & 1,08 & $27,53^{* *}$ \\
\hline Afecto 3 & 2,91 & 1,29 & 3,24 & 1,30 & 2,56 & 1,19 & $27,69^{* *}$ \\
\hline Afecto 4 & 3,64 & 1,19 & 3,94 & 1,09 & 3,31 & 1,20 & $29,67^{* *}$ \\
\hline Afecto 5 & 2,83 & 1,28 & 3,12 & 1,37 & 2,52 & 1,09 & $21,49^{* * *}$ \\
\hline Intención 1 & 4,26 & 0,98 & 4,48 & 0,89 & 4,03 & 1,03 & $20,85^{* *}$ \\
\hline Intención 2 & 4,29 & 0,84 & 4,58 & 0,67 & 3,99 & 0,89 & $51,59^{* *}$ \\
\hline Intención 3 & 4,32 & 0,83 & 4,49 & 0,79 & 4,14 & 0,84 & $16,85^{* *}$ \\
\hline Intención 4 & 3,90 & 1,08 & 4,25 & 0,95 & 3,53 & 1,09 & $47,42^{* *}$ \\
\hline Intención 5 & 3,82 & 1,16 & 3,90 & 1,24 & 3,73 & 1,07 & 2,042 \\
\hline Intención 6 & 3,89 & 1,09 & 4,06 & 1,09 & 3,71 & 1,06 & $9,901^{* *}$ \\
\hline Intención 7 & 2,72 & 1,20 & 2,77 & 1,35 & 2,66 & 1,03 & 0,772 \\
\hline
\end{tabular}

\section{ANÁLISIS Y RESULTADOS}

\subsection{Análisis de la fiabilidad, dimensionalidad y validez de las escalas de medida}

El estudio preliminar de la dimensionalidad de las escalas se realizó a través de un análisis factorial exploratorio de componentes principales con rotación Varimax (Hair et $a l .$, 1999), usando el criterio de los autovalores mayores a la unidad. Todas las escalas, a excepción de la de intenciones de comportamiento de queja, resultaron unidimensionales.

La escala de insatisfacción $(\alpha=0,854)$ explica una variabilidad total del $64,76 \%$ y la escala de afectos negativos $(\alpha=0,772)$ arroja una variabilidad de $52,62 \%$. En cuanto a la escala multidimensional de intenciones de comportamiento de queja extrae tres dimensiones: 1) intenciones de respuestas privadas (intención 1-2-3-4, $\alpha=0,754$ ), 2) intenciones de respuestas de queja (intención 5-6, $\alpha=0,784$ ) e 3 ) intenciones de respuestas a terceras partes (intención 7); explicando el 62,05\% de la variabilidad total. Estos resultados reflejaron valores satisfactorios sobre la dimensionalidad de las escalas, ya que la varianza explicada supera el umbral de $50 \%$ y existe buena consistencia interna según los valores de fiabilidad superiores a 0,7 (Hair et al., 1999). 
Esta propuesta de dimensionalidad quedó confirmada tras la aplicación de un análisis factorial confirmatorio con el software EQS 6.1. La estimación del modelo de medida, de segundo orden en el caso de la escala multidimensional de las intenciones de comportamiento de queja, se llevó a cabo mediante el método de verosimilitud robusto (Bentler, 1995), utilizando como entrada la matriz de covarianzas (Jöreskog y Sörbom, 1996). Tal como se recoge en la tabla 4 el ajuste del análisis resulta aceptable -RMSEA inferior a 0,1 y los índices GFI, CFI, AGFI presentan valores elevados próximos a 1 (Hair et al., 1999).

El análisis de la validez de constructo de las escalas se efectuó estudiando la validez convergente y divergente. La validez convergente recoge el grado en que dos o más intentos de medir el mismo concepto están de acuerdo entre sí. Para medir esta relación en factores unidimensionales, se establece que las variables poseen ponderaciones significativas y elevadas (Anderson y Gerbing, 1988). Así, dicha validez se comprobó a partir de las cargas estandarizadas correspondientes a cada una de las dimensiones y subdimensiones (Tabla 4). Las saturaciones resultaron en su totalidad superiores a 0,5 y todos los valores asociados del estadístico t-Student significativos al nivel 1\%. Con ello, podemos concluir que las escalas están dotadas de validez convergente (Steemkamp y Van Trijp, 1991).

Tabla 4

Modelo de medida. Dimensionalidad, fiabilidad y validez de las escalas

\begin{tabular}{|l|l|c|}
\cline { 2 - 3 } \multicolumn{5}{c|}{} & \multicolumn{1}{c|}{ Ítems } & \multicolumn{1}{c|}{$\begin{array}{c}\text { Cargas factoriales } \\
\text { estandarizadas }\end{array}$} \\
\hline \multirow{5}{*}{ Insatisfacción } & Insatisfacción 1 & 0,660 \\
\cline { 2 - 3 } & Insatisfacción 2 & $0,873\left(16,78^{* *}\right)$ \\
\cline { 2 - 3 } & Insatisfacción 3 & $0,833(13,67 * *)$ \\
\cline { 2 - 3 } & Insatisfacción 4 & $0,705(10,89 * *)$ \\
\cline { 2 - 3 } & Insatisfacción 5 & $0,763\left(13,88^{* *}\right)$ \\
\cline { 2 - 3 } & Fiabilidad compuesta & $87,86 \%$ \\
\cline { 2 - 3 } & Varianza extraída & $63,36 \%$ \\
\hline \multirow{5}{*}{ Aesconfirmación de expectativas } & Desconfirmación & 1,00 \\
\hline \multirow{5}{*}{ Afectos Negativos } & Afecto 1 & 0,742 \\
\cline { 2 - 3 } & Afecto 2 & $0,801(8,59 * *)$ \\
\cline { 2 - 3 } & Afecto 3 & $0,657(8,35 * *)$ \\
\cline { 2 - 3 } & Afecto 4 & $0,685(8,68 * *)$ \\
\cline { 2 - 3 } & Afecto 5 & $0,886(8,23 * *)$ \\
\cline { 2 - 3 } & Fiabilidad compuesta & $84,67 \%$ \\
\cline { 2 - 3 } & Varianza extraída & $50,24 \%$ \\
\hline & & \\
\hline
\end{tabular}




\begin{tabular}{|c|c|c|}
\hline & \\
\hline & Ítems & $\begin{array}{c}\text { Cargas factoriales } \\
\text { estandarizadas }\end{array}$ \\
\hline \multirow{6}{*}{ Dimensión 1: Intenciones de respuestas privadas } & Intención 1 & 0,771 \\
\hline & Intención 2 & $0,671(7,28 * *)$ \\
\hline & Intención 3 & $0,654(7,38 * *)$ \\
\hline & Intención 4 & $0,709(8,52 * *)$ \\
\hline & Fiabilidad compuesta & $81,84 \%$ \\
\hline & Varianza extraída & $50,75 \%$ \\
\hline \multirow{4}{*}{ Dimensión 2: Intenciones de respuestas de queja } & Intención 5 & 0,850 \\
\hline & Intención 6 & $0,816\left(10,75^{* *}\right)$ \\
\hline & \begin{tabular}{|l|} 
Fiabilidad compuesta \\
\end{tabular} & $81,96 \%$ \\
\hline & Varianza extraída & $66,59 \%$ \\
\hline $\begin{array}{l}\text { Dimensión 3: Intenciones de respuestas a } 3^{a s} \\
\text { partes }\end{array}$ & Intención 7 & 1,00 \\
\hline \multicolumn{3}{|c|}{$\begin{array}{l}\left.\text { Medidas de ajuste del modelo de medida global: } \chi^{2} \text { Satorra-Bentler }(\mathrm{g} .1)=129\right)=292.99 \text { (p- } \\
\text { value }=0.000) ; \text { RMSEA }=0.061 ; \text { CFI }=0.925 ; \text { BB-NFI }=0.877 \text {; BB-NNFI }=0.907 ; \text { GFI }=0,923 \text {; } \\
\text { AGFI }=0,907\end{array}$} \\
\hline \multicolumn{3}{|c|}{ **: Valores del estadístico t entre paréntesis $(\mathrm{p}$-valor $<0,01)$} \\
\hline
\end{tabular}

En cuanto al análisis de la validez discriminante, comprobación de que cada factor representa una dimensión separada, se llevó a cabo a través de las correlaciones lineales, o covarianzas estandarizadas, entre los factores latentes. Estos valores muestran indicios de validez discriminante ya que toman valores alejados de la unidad, resultando en todos los casos, indicativo de discriminación entre las diferentes dimensiones analizadas (Bagozzi, 1994). Una vez elevadas al cuadrado, resultan menores que la cantidad de varianza extraída, por lo que podemos garantizar la validez discriminante de las variables latentes. Para analizar en profundidad esta tipo validez se comprobó que el intervalo de confianza de la correlación entre cada par de factores latentes no contiene el valor 1 (Tabla 5), demostrando que dichos factores representan conceptos notablemente diferentes (Anderson y Gerbing, 1988).

Tabla 5

Validez discriminante de las escalas

\begin{tabular}{|l|c|c|}
\cline { 2 - 3 } \multicolumn{1}{c|}{} & Correlacion $^{2}$ & IC $(95 \%)$ \\
\hline Insatisfacción-Afectos negativos & 0,073 & {$[0,009 ; 0,137]$} \\
\hline Insatisfacción-Intenciones de comportamientos de queja & 0,465 & {$[0,355 ; 0,575]$} \\
\hline Insatisfacción-Desconfirmación de expectativas & 0,216 & {$[0,128 ; 0,304]$} \\
\hline Afectos negativos-Intenciones de comportamientos de queja & 0,134 & {$[0,056 ; 0,212]$} \\
\hline Afectos negativos-Desconfirmación de expectativas & 0,163 & {$[0,089 ; 0,237]$} \\
\hline $\begin{array}{l}\text { Intenciones de comportamientos de queja-Desconfirmación } \\
\text { de expectativas }\end{array}$ & 0,246 & {$[0,134 ; 0,358]$} \\
\hline
\end{tabular}




\subsection{Estimación del modelo causal}

Tras comprobar la fiabilidad y validez de las escalas propuestas, procedimos a contrastar las hipótesis planteadas a través del análisis de un modelo causal en la totalidad de individuos. Los resultados de la estimación del modelo quedan reflejados en la figura 2. La calidad del ajuste, analizado mediante diferentes medidas de bondad, es aceptable. Teniendo en cuenta la matización sobre el contraste Chi-cuadrado (Bentler y Bonnet, 1980; James et al., 1982) ${ }^{4}$, podemos concluir que el modelo causal presenta un ajuste satisfactorio (Tabla 6).

Tabla 6

\section{Indicadores de ajuste del modelo estructural}

\begin{tabular}{|c|c|c|}
\hline Indicadores de bondad de ajuste & Nivel óptimo recomendado & Valor del modelo causal \\
\hline$\chi^{2}$ Sat-B. (p-valor) & p-valor $>0,05$ & $\begin{array}{c}\chi^{2} \text { Sat-B. }(\mathrm{g} .1 .=140)=295.29(\mathrm{p}- \\
\text { valor }=0.000)\end{array}$ \\
\hline RMSEA & $<0,08$ & 0,061 \\
\hline GFI & Alto, cercano a 1 & 0,909 \\
\hline AGFI & Alto, cercano a 1 & 0,897 \\
\hline BB-NNFI & Alto, cercano a 1 & 0,907 \\
\hline BB-NIFI & Alto, cercano a 1 & 0,876 \\
\hline $\mathrm{CFI}$ & Alto, cercano a 1 & 0,924 \\
\hline
\end{tabular}

Figura 2

Modelo estructural de las relaciones causales entre las variables



${ }^{4}$ Diversos autores han señalado los problemas de este estadístico para la evaluación del modelo en relación con el tamaño muestral. Debido a esta matización, hemos contemplado otros índices de bondad de ajuste global. 
Haciendo referencia a los coeficientes estimados entre los factores latentes del modelo, los resultados indican la existencia de relaciones significativas entre las variables. En cuanto a las causas de la insatisfacción, por una parte, podemos afirmar que la desconfirmación de expectativas influye positivamente y de manera significativa sobre la insatisfacción y sobre los afectos negativos. Por tanto, se puede verificar las $\mathrm{H}_{1}$ y $\mathrm{H}_{3}$ planteadas en el estudio. Estos resultados se encuentran en la misma línea que los obtenidos en diversas investigaciones aplicadas en el ámbito de los servicios (Menon y Dubé, 2000; Bigné y Andreu, 2002). Por otra parte, los coeficientes estimados en el modelo revelan que los afectos negativos no influyen significativamente en la variable insatisfacción, no pudiéndose confirmar la $\mathrm{H}_{2}$. Por tanto, el cumplimiento de las hipótesis planteadas en este trabajo permite confirmar, en nuestro contexto de aplicación, la naturaleza cognitiva de la insatisfacción que plantea el enfoque tradicional sobre la formación de la satisfacción de Oliver (1980).

Respecto a las consecuencias de la insatisfacción, los coeficientes estimados del modelo muestran que dicha insatisfacción presenta una carga significativa y positiva sobre las intenciones de comportamientos de queja, por lo que se cumpliría también la $\mathrm{H}_{4}$. Además, el análisis de la dimensionalidad de las escalas ha revelado la existencia de tres dimensiones en la escala de intenciones (Tabla 4). Este carácter multidimensional se ha reconocido teórica y empíricamente en la mayoría de investigaciones sobre comportamiento de queja (Liu y McClure, 2001). Y también existen evidencias empíricas sobre la influencia de la insatisfacción sobre las intenciones posteriores (Mittal y Kamakura, 2001; Oh, 2006).

\subsection{Resultados del análisis multimuestra}

Tras los análisis del modelo causal para la totalidad de la muestra, pasamos a contrastar el papel moderador que desempeña la atribución externa. De forma general, un moderador es una variable cualitativa o cuantitativa que afecta a la dirección o a la intensidad de la relación entre una variable predictora o independiente y una variable dependiente o criterio (Baron y Kenny, 1986; p. 1174). En nuestro escenario de investigación, consideramos como una forma adecuada de proceder, distinguir entre aquellos individuos con elevado nivel de atribuciones externas $(\mathrm{N}=194)$ y aquellos que tienen bajo nivel de atribuciones externas ( $\mathrm{N}=186)$ (Tabla 3). Esta distinción nos permitirá medir el efecto de las variables antecedentes en la variable dependiente a través de los parámetros estimados de las relaciones causales y entonces testar la diferencia entre dichos coeficientes -gamma y beta en notación LISREL- utilizando un test de moderación adecuado (Baron y Kenny, 1986). El análisis multimuestra ha sido efectuado siguiendo las recomendaciones metodológicas propuestas por Jaccard y Wan (1996).

El primer paso consiste en la estimación por separado de los parámetros que definen las diferentes relaciones causales en cada uno de los dos grupos, así como una medida de bondad de ajuste del modelo global para ambos grupos simultáneamente. Esta solución multigrupo aparece recogida en la tabla 7. 
Tabla 7

Análisis multigrupo: relaciones causales en función del nivel de atribución externa

\begin{tabular}{|c|c|c|}
\hline & $\begin{array}{l}\text { Alto nivel de atribuciones } \\
\qquad(\mathrm{N}=194)\end{array}$ & $\begin{array}{l}\text { Bajo nivel de atribuciones } \\
\qquad(\mathrm{N}=186)\end{array}$ \\
\hline & Carga estand. (t-valor) & Carga estand. (t-valor) \\
\hline Desconfirmación $\rightarrow$ Afectos negativos & $0,329(3,33 * *)$ & $0,226\left(2,55^{*}\right)$ \\
\hline Desconfirmación $\rightarrow$ Insatisfacción & $0,432(5,82 * *)$ & $0,326(4,03 * *)$ \\
\hline Afectos negativos $\rightarrow$ Insatisfacción & $0,043(0,494)$ & $0,121(1,31)$ \\
\hline $\begin{array}{l}\text { Insatisfacción } \rightarrow \text { Intenciones Comp. } \\
\text { Queja }\end{array}$ & $0,650(5,21 * *)$ & $0,669(4,93 * *)$ \\
\hline \multicolumn{3}{|l|}{ Índices de ajuste } \\
\hline \multicolumn{3}{|c|}{$\begin{array}{l}\left.\text { Chi }^{2} \text { Sat. }(\mathrm{g} .1 .=260)=469,13 \text { (p-valor }=0,000\right) ; \text { RMSEA }=0,060 ; \text { CFI=0,890; BB-NFI=0,869; BB- } \\
\text { NNFI=0,899; GFI LISREL = 0,848; AGFI LISREL = 0,819 }\end{array}$} \\
\hline \multicolumn{3}{|c|}{ **Significativo al 0,$01 ; *$ : Significativo al 0,05} \\
\hline
\end{tabular}

El análisis multimuestra indica que las relaciones causales entre ambos grupos no se manifiestan de la misma manera, lo cual puede poner de manifiesto el papel moderador del grado de atribución. Tal como se muestra en la tabla 7, las estimaciones causales de la desconfirmación sobre la insatisfacción y sobre los afectos se manifiestan ligeramente más fuertes en el grupo de clientes que presentan un nivel elevado de atribución externa, mientras que el efecto de la insatisfacción sobre las intenciones de comportamientos de queja es ligeramente superior entre los clientes con baja atribución externa.

Con el fin de comprobar si existen diferencias significativas entre las estimaciones de los parámetros causales, volvimos a estimar el modelo planteado, introduciendo la restricción como hipótesis nula que establece que los coeficientes de regresión en el modelo estructural son iguales en los dos grupos (Iglesias y Vázquez, 2001). En esta segunda etapa, y gracias a la prueba del multiplicador de Lagrange (lmtest), se pueden observar diferencias significativas entre los parámetros de ambas submuestras. Es decir, podemos comprobar si la eliminación de las restricciones produce un cambio significativo en el estadístico $\mathrm{Chi}^{2}$, lo que conllevaría a rechazar la restricción de igualdad en los parámetros ya que su eliminación mejoraría significativamente el ajuste del modelo (véase tabla 8).

Tabla 8

Contraste de hipótesis en función del nivel de atribución externa

\begin{tabular}{lcccc}
\hline \multicolumn{1}{c}{ Restricciones } & gl & Diferencias & $\chi^{2}$ & p-valor \\
\hline Desconfirmación $\rightarrow$ Afectos negativos & 1 & 0,701 & 0,403 \\
Desconfirmación $\rightarrow$ Insatisfacción & 1 & 1,943 & 0,163 \\
Afectos negativos $\rightarrow$ Insatisfacción & 1 & 0,039 & 0,844 \\
Insatisfacción $\rightarrow$ Intenciones Comp. Queja & 1 & 0,020 & 0,887 \\
\hline
\end{tabular}


El estadístico asociado a la diferencia de parámetros para cada una de las restricciones por separado indica que la eliminación de las restricciones no mejora el ajuste del modelo. Por tanto, podemos afirmar que el grado de atribución externa no modera de manera significativa las causas y consecuencias de la insatisfacción.

\section{CONCLUSIONES}

Según los objetivos de este trabajo, pretendíamos estudiar las causas y consecuencias de la insatisfacción analizando las relaciones causales entre la desconfirmación de expectativas, los afectos negativos, la insatisfacción y las intenciones de comportamientos de queja. La estimación del modelo causal ofrece resultados importantes para conocer el componente cognitivo y afectivo de la insatisfacción, así como las respuestas no conductuales de los consumidores expresadas en forma de intenciones de comportamientos de queja.

Respecto a la causas de la insatisfacción, la desconfirmación de expectativas se revela como una variable que tiene un efecto directo y positivo sobre los juicios de insatisfacción, lo cual se encuentra en la línea de numerosas investigaciones que abordan el estudio de la formación de la satisfacción a partir de la desconfirmación (Bigné y Andreu, 2002). Además, la desconfirmación influye en la intensidad de los afectos negativos. Sin embargo, estos afectos no tienen una influencia significativa en el nivel de insatisfacción experimentado, por lo que no podemos confirmar que exista un efecto indirecto de la desconfirmación a través de los afectos negativos. Por tanto, en nuestro contexto de aplicación, la insatisfacción de consumidores que realizan atribuciones externas, es decir, culpabilizan al restaurante de la situación, tiene mayor naturaleza cognitiva que afectiva, ya que la valoración cognitiva basada en la desconfirmación es la que se ha revelado como causante de la insatisfacción. Estos resultados se encuentran en la línea de trabajos que confirman el efecto de la desconfirmación de expectativas sobre el nivel de satisfacción del consumidor (Oliver et al., 1997; Roch y Poister 2006; James, 2007).

Respecto a las consecuencias de la insatisfacción, estos juicios se muestran claramente como una causa de las intenciones de comportamientos de queja de los individuos que realizan atribuciones externas. Los resultados indican la existencia de tres dimensiones que se manifiestan en intenciones de respuestas privadas, de queja y a terceras partes (Liu y McClure, 2001). También existen evidencias empíricas anteriores sobre la relación causal entre niveles de satisfacción e intenciones de comportamientos (Choi et al., 2002). Por tanto, podemos concluir que los sujetos que asignan en mayor o menor medida al restaurante la culpa de la insatisfacción muestran claramente intenciones de desarrollar tanto acciones de queja al proveedor y a terceras partes, como acciones de carácter privado.

Por último, el análisis de las relaciones causales en cada una de las submuestras obtenidas según el grado de atribuciones externas permite concluir que las diferencias en el efecto de las variables no son significativas. Esto implicaría que, en nuestro contexto de análisis, las atribuciones externas no ejercen un papel moderador en el estudio de las causas y consecuencias de la insatisfacción. Por tanto, con independencia del nivel de atribuciones que realicen los consumidores, éstos utilizarán los mismos criterios en la valoración de su experiencia para emitir sus juicios de insatisfacción y tendrán similares intenciones de respuestas de queja posteriores. 
Trasladando estos resultados a la práctica empresarial, los responsables de la gestión del negocio de los restaurantes deben ser conscientes de la importancia que tiene no sólo cumplir las expectativas del cliente sino también superarlas. Esto permitiría conseguir una desconfirmación positiva y, por tanto, evitar la insatisfacción, y también reducir los afectos negativos, sobre todo, los afectos relativos al enfado y la indignación. A pesar de que los resultados han mostrado que los afectos negativos no influyen de forma significativa sobre la insatisfacción, si que se ha revelado la desconfirmación como un antecedente importante de los afectos. Por tanto, la comparación que realizan los individuos entre la percepción de los resultados y sus expectativas es un claro determinante de sus respuestas afectivas. Además, también estos resultados ponen de manifiesto una vez más la necesidad de implantar un programa adecuado de tratamiento de la insatisfacción y las quejas (Davidow, 2003), debido a sus efectos sobre las intenciones de comportamientos posteriores. Concretamente, los individuos insatisfechos muestran una elevada predisposición a desarrollar acciones de queja, ya sea al restaurante o a organismos de protección del consumidor, conductas de cambio y de difusión de la insatisfacción a través de comentarios boca-oreja negativos. Aunque el nivel de satisfacción que necesita un individuo para expresar una intención es diferente al nivel necesario para desarrollar un determinado comportamiento (Mittal y Kamakura, 2001; p.135), los resultados de este trabajo indican que se debe prestar especial atención a las respuestas no conductuales, como las actitudes o intenciones, por su posible contribución a largo plazo en los comportamientos de queja.

Las propuestas de investigación que sugerimos se encuentran en la línea de profundizar en el proceso de formación de la insatisfacción y sus respuestas posteriores. Para avanzar en este estudio, sugerimos la aplicación del modelo causal estimado en otros servicios con alto potencial para generar situaciones insatisfactorias, con el fin de conocer si se mantienen las mismas relaciones con la desconfirmación, los afectos y las intenciones de comportamientos posteriores. Según los datos sobre la evolución de las consultas y reclamaciones por sectores económicos del Instituto Nacional de Consumo (2007), las comunicaciones electrónicas, los servicios financieros y la vivienda en propiedad son los sectores que encabezan la lista en cuanto a número de quejas atendidas, por lo que consideramos ámbitos de interés para su estudio por sus elevados índices de insatisfacción.

A nivel conceptual, también sería conveniente añadir otros procesos cognitivos que permitieran completar el origen de la insatisfacción, como pueden ser los juicios de equidad, la desconfirmación de deseos y normas basadas en la experiencia o las dimensiones de las atribuciones. Respecto a este último enfoque, en nuestro trabajo se ha considerado la atribución externa como una variable para segmentar a los sujetos objeto de estudio. Dado que dicha variable no ha resultado ser moderadora en las relaciones causales, podría ser interesante continuar profundizando en su contribución a través de otra metodología de segmentación, como el análisis Cluster o la metodología CHAID (Magidson, 1994). También se podría avanzar en esta línea analizando el papel mediador de las atribuciones externas en la formación de la insatisfacción. Por ejemplo, se podría utilizar la metodología empleada en el trabajo de Maxham III y Netemeyer (2003) que, aplicando la regresión con mediación, investiga los efectos mediadores de algunos determinantes de la satisfacción e intenciones conductuales.

Respecto al análisis de las respuestas a la insatisfacción, en futuras investigaciones se podría ampliar la formación de las intenciones de los comportamientos de queja examinan- 
do la contribución de variables relacionadas con la situación insatisfactoria como la probabilidad de éxito de la queja (Morel et al., 1997) o la importancia de la situación (Stephens y Gwinner, 1998). Del mismo modo, sería interesante contemplar el efecto de otras variables del consumidor ajenas a la experiencia de insatisfacción, tales como sus actitudes hacia la queja (Singh y Wilkes, 1996) o su nivel de información y experiencia (Martín, 1991). También se podría analizar la relación de las intenciones de comportamientos de queja con factores de corte socio-demográfico (Phau y Sari, 2004), o de tipo psicográfico estudiando la relación que puede tener personalidad del individuo (Badey y Grace, 2006).

\section{REFERENCIAS BIBLIOGRÁFICAS}

ANDERSON, J.C. Y GERBING, D.W. (1988): "Structural equation modeling in practice: a review and recommended two-step approach", Psychological Bulletin, Vol. 103, No. 3, pp. 411-423.

ANDERSON, E.W. Y SULLIVAN, M.W. (1993): "The antecedents and consequences of customer satisfaction for forms", Marketing Science, Vol. 12, No. 2, pp. 125-143.

ANDREASSEN, T.W. (2000): “Antecedents to satisfaction with service recovery”, European Journal of Marketing, Vol. 34, No. 1/2, pp. 156-175.

ANDREU, L. (2003): "Emociones del consumidor: componentes y consecuencias en marketing", Estudios sobre Consumo, Vol. 64, No. 1, pp. 9-25.

BABIN, B.J.; GRIFFIN, M. Y BABIN, L. (1994): "The effect of motivation to process on consumers' satisfaction reactions", Advances in Consumer Research. Alle, C.T. y Roedder, D. (eds.), Provo, UT: Association for Consumer Research.

BADEY, K. Y GRACE, D. (2006): "Segmenting service complainers and non-complainers on the basis of consumer characteristics", Journal of Services Marketing, Vol. 20, No. 3, pp. 178-187.

BAGOZZI, R. (1994): "Structural Equation Model in Marketing Research", Basic Principles, Principles of Marketing Research. Blackwell Publishers, Oxford.

BAGOZZI, R.P.; GOPINATH, M. Y NYER, P.U. (1999): “The Role of Emotions in Marketing”, Journal of the Academy of Marketing Science, Vol. 27, No. 2, pp. 184-206.

BARON, R.M. Y KENNY, D.A. (1986): "The moderator-mediator variable distinction in social psychological research: conceptual, strategic, and statistical considerations", Journal of Personality and Social Psychology, Vol. 51, No. 6, pp. 1173-1182.

BENTLER, P. (1995): EQS Structural Equations Program Manual, Encino, Multivariate Software.

BENTLER, P.M. Y BONNET, D.G. (1980): "Significance test and goodness of fit in the analysis of covariances structures", Psychological Bulletin, Vol. 88, pp. 588-606.

BIGNÉ, J.E. Y ANDREU, L. (2002): "Análisis de la satisfacción en la experiencia del consumidor: una aplicación en museos interactivos", XIV Encuentro de Profesores Universitarios de Marketing, Granada, septiembre, pp. 497-510.

BIGNÉ, J.E. Y ANDREU, L. (2004): "Modelo cognitivo-afectivo de la satisfacción en servicios de ocio y turismo", Cuadernos de Economía y Dirección de Empresas, No. 21, pp. 89-120.

BOUGIE, R.; PIETERS, R. Y ZEELENBERG, M. (2003): "Angry customers don't come back, they get back: The experience and behavioural implications of anger and dissatisfaction in services", Journal of the Academy of Marketing Science, Vol. 31, No. 4, pp. 377-393.

BOWEN, D. (2001): "Antecedents of consumer satisfaction and dissatisfaction (CS/D) on long haul inclusive tours: a reality check on theoretical considerations", Tourism Management, Vol. 22, No. 1, pp. 49-61.

CADOTTE, E.R.; WOODRUFF, R.B. Y JENKINS, R.L. (1987): "Expectations and norms in models of consumer satisfaction”, Journal of Marketing Research, Vol. 24, pp. 305-314. 
CASADO, A.B.; MAS, F. Y AZORÍN, A. (2004): “Atribución, satisfacción, calidad percibida e intención de conducta en encuentros de servicio fracasados", Revista Europea de Dirección y Economía de la Empresa, Vol. 13, No. 1, pp. 45-62.

CHEBAT, J.C. Y MICHON, R. (2003): "Impact of ambient odors on mall shoppers' emotions, cognition and spending", Journal of Business Research, Vol. 56, No. 7, pp. 529-539.

CHOI, K.; CHO, W.H.; LEE, S.; LEE, H. Y KIM, C. (2002): "The relationship among quality, value, satisfaction and behavioral intention in health care provider choice: a South Korean study", Journal of Business Research, Vol. 57, No. 8, pp. 913-921.

EKINCI, Y.; DAWES, P.L. Y MASSEY, G.R. (2008): "An extended model of the antecedents and consequences of consumer satisfaction for hospitality services", European Journal of Marketing, Vol. 42, No. 1/2, pp. 35-68.

EREVELLES, S. Y LEAVITT, C. (1992): "A comparison of current models of consumer satisfaction/dissatisfaction", Journal of Consumer Satisfaction, Dissatisfaction and Complaining Behavior, Vol. 5, pp. 104-114.

ESTEBAN, A.; MILLÁN, A. Y MARTÍN-CONSUEGRA, D. (2002): "Análisis de la satisfacción en los servicios de los supermercados desde la perspectiva de los consumidores y detallistas", XIV Encuentro de Profesores Universitarios de Marketing, Granada, pág. 481-496.

FOLKES, V.S. (1984): "Consumer reactions to product failure: an attributional approach", Journal of Consumer Research, Vol. 10, pp. 398-409.

GIESE, J.L. Y COTE, J.A. (2000): "Defining consumer satisfaction”, Academy of Marketing Science Review, Vol. 1, pp. 1-34.

HAIR, J.F.JR.; ANDERSON, R.E; TATHAM, R.L. Y BLACK, W.C. (1999): Análisis Multivariante. $5^{\text {a }}$ Ed. Prentice Hall, Londres.

INSTITUTO NACIONAL DE CONSUMO (2007): Consultas y reclamaciones en las organizaciones de consumidores de ámbito estatal presentes en el consejo de consumidores y usuarios: 2007. www.consumo-inc.es.

IZARD, C.E. (1977): Human Emotions. New York, Plenum Press.

IZARD, C.E. (1991): The Psychology of Emotions. New York: Plenum.

IGLESIAS, V. Y VÁZQUEZ, R. (2001): "The moderating Effects of Exclusive Dealing Agreements on distributor Satisfaction", Journal of Strategic Marketing, Vol. 9, No. 3, pp. 215-231.

JACCARD, J. Y WAN, C.K. (1996): Lisrel Approach to Interaction Effects in Multiple Regression. Thousand Oaks, CA: Sage Publications.

JAMES, O. (2007): "Evaluating the expectations disconfirmation and expectations anchoring approaches to citizen satisfaction with local public services", Journal of Public Administration Research and Theory, Vol. 19, No. 1, pp. 107-123.

JOHNSON, M.D.; ANDERSON, E.W. Y FORNELL, C. (1995): "Rational and adaptative performance expectations in a customer satisfaction framework", Journal of Consumer Research, Vol. 21, pp. 695-707.

JÖRESKOG, K.G. Y SÖRBOM, D. (1996): PRELIS 2: User's Reference Guide. Scientific Software International.

JUN, S.; HYUN, Y.J.; GENTRY, J.W. Y SONG, C. (2001): "The relative influence of affective experience on consumer satisfaction under versus negative discrepancies", Journal of Consumer Satisfaction, Dissatisfaction and Complaining Behavior, Vol. 14, pp. 141-153.

KATTARA, H.S.; WEHEBA, D. Y EL-SAID O.A. (2008): "The impact of employee behaviour on customers' service quality perceptions and overall satisfaction", Tourism and Hospitality Research, Vol. 8, No. 4, pp. 309-323.

LAZARUS, R.S. (1991): Emotion and Adaptation. New York: Oxford University Press.

LILJANDER, V. Y STRANDVIK, T. (1997): "Emotions in service satisfaction", International Journal of Service Industry Management, Vol. 8, No. 2, pp. 148-169. 
LIU, R.R. Y MCCLURE, P. (2001): "Recognizing cross-cultural differences in consumer complaint behavior and intentions: an empirical examination", Journal of Consumer Marketing, Vol. 18, No. 1, pp. 54-74.

MAGIDSON, J. (1994): The CHAID Approach to Segmentation Modeling: Chi-Square Automatic Interaction Detection. In Advanced Method of Marketing Research. Ed. R. Bagozzi. Blackwell. Cambridge.

MANO, H. Y OLIVER, R.L. (1993): "Assessing the dimensionality and structure of the consumption experience: evaluation, feeling and satisfaction", Journal of Consumer Research, Vol. 20, pp. 451-466.

MATTILA, A.S. Y RO, H. (2007): "Discrete negative emotions and customer dissatisfaction responses in a casual restaurant setting", Journal of Travel \& Tourism Marketing, Vol. 26, No. 2, pp. 120.

MATTILA, A.S. Y RO, H. (2008): "Discrete negative emotions and customer dissatisfaction responses in a casual restaurant setting", Journal of Hospitality \& Tourism Research, Vol. 32, No. 1, pp. 89-107.

MAUTE, M.F. Y DUBÉ, L. (1999): "Patterns of emotional responses and behavioural consequences of dissatisfaction", Applied Psychology: An International Review, Vol. 48, No. 3, pp. 349-366.

MAXHAM III, J.G. Y NETEMEYER, R.G. (2002): "A longitudinal study of complaining customers' evaluations of multiple service failures and recovery efforts", Journal of Marketing, Vol. 66, pp. 57-71.

MAXHAM III, J.G. Y NETEMEYER, R.G. (2003): "Firms reap what they sow: the effects of shared values and perceived organizational justice on customer's evaluations of complaint handling”, Journal of Marketing, Vol. 67, pp. 46-62.

MITTAL, V. Y KAMAKURA, W.A. (2001): "Satisfaction, repurchase intent and repurchase behavior: investigating the moderating effect of consumer characteristics", Journal of Marketing Research, Vol. 38, No. 1, pp. 131-142.

MITTAL, V.; KUMAR, P.J. Y TSIROS, M. (1999): "Attribute-level performance, satisfaction and behavioral intentions over time: a consumption-system approach", Journal of Marketing, Vol. 63, pp. 88-101.

MOREL, K.P.N.; PIESZ, T.B.C. Y WILKE, H.A.M. (1997): "Motivation, capacity and opportunity to complain: towards a comprehensive model of consumer complaint behavior", Advances in Consumer Research, Vol. 24, pp. 464-469.

OH, D.G. (2006): "Complaining intentions and their relationships to complaining behavior of academic library users in South Korea", Library Management, Vol. 27, No. 3, pp. 168-189.

OLIVER, R.L. (1980): “A cognitive model of the antecedents and consequences of satisfaction decisions", Journal of Marketing Research, Vol. 17, pp. 460-469.

OLIVER, R.L. (1981): "Measurement and evaluation of satisfaction processes in retail settings", Journal of Retailing, Vol. 57, No. 3, pp. 25-48.

OLIVER, R.L. (1993): "Cognitive, affective and attribute bases of the satisfaction response", Journal of Consumer Research, Vol. 20, pp. 418-430.

OLIVER, R.L. Y DESARBO, W.S. (1988): "Response determinants in satisfaction judgements", Journal of Consumer Research, Vol. 14, pp. 495-507.

OLIVER, R.L.; RUST, R.T. Y VARKI, S. (1997): "Customer delight: foundations, findings and managerial insight", Journal of Retailing, Vol. 73, No. 3, pp. 311-336.

ONYEASO, G. (2007): “Are customers' dissatisfaction and complaints behaviours positively related? Empirical tests. Journal of American Academy of Business, Vol. 11, No. 1, pp. 18-24.

ORTONY, A.; CLORE, G.L. Y COLLINS, A. (1988): The Cognitive Structure of Emotions. Cambridge: Cambridge University Press.

PHAU, I. Y SARI, R.P. (2004): "Enganging in complaint behaviour", Marketing Intelligence \& Planning, Vol. 22, No. 4, pp. 407-426. 
PRAKASH, V. (1991): "Intensity of dissatisfaction and consumer complaint behaviors", Journal of Consumer Satisfaction, Dissatisfaction and Complaining Behavior, Vol. 4, pp. 110-122.

ROCH, C.H. Y POISTER, T.H. (2006): "Citizens, accountability, and service satisfaction", Urban Affaire Review, Vol. 41, pp. 292-308.

ROOS, I. (2002): "Methods of investigating critical incidents", Journal of Service Research, Vol. 4, No. 3, pp. 193-204.

SINGH, J. (1990): "Voice, exit and negative word-of-mouth behaviors: an investigation across three service categories", Journal of the Academy of Marketing Science, Vol. 18, No. 1, pp. 1-15.

SINGH, J. Y WILKES, R.E. (1996): "When consumers complain: a path analysis of the key antecedents of complaint response estimates", Journal of the Academy of Marketing Science, Vol. 24, No. 4, pp. 350-365.

SMITH, A.K. Y BOLTON, R.N. (2002): "The effect of consumers' emotional responses to service failures on their recovery effort evaluations and satisfaction judgments", Journal of Academy of Marketing Science, Vol. 30, pp. 5-24.

SPRENG, R.A.; MACKENZIE, S.B. Y OLSHAVSKY, R.W. (1996): "A reexamination of the determinants of consumer satisfaction", Journal of Marketing, Vol. 60, pp. 15-32.

STEEMKAMP, E.M. Y VAN TRIJP, C.M. (1991): "The Use of LISREL in Validating Marketing Constructs", International Journal of Research in Marketing, Vol. 8, pp. 283-299.

STEPHENS, N. Y GWINNER, K.P. (1998): "Why don't some people complain? A cognitive-emotive process model of consumer complaint behavior", Journal of the Academy of Marketing Science, Vol. 26, No. 3, pp. 172-189.

SWANSON, S.R. Y KELLEY, S.W. (2001): "Service recovery attributions and word-of-mouth intentions", European Journal of Marketing, Vol. 35, No. 1/2, pp. 194-211.

DAVIDOW, M. (2003): "Organizational responses to customer complaints: what works and what doesn't", Journal of Service Research, Vol. 5, No. 3, pp. 225-250.

THENG, G. Y NG, S. (2001): "Individual and situational factors influencing negative word-ofmouth behavior", Canadian Journal of Administrative Sciences, Vol. 18, No. 3, pp. 163-178.

TSIROS, M.; MITTAL, V. Y ROSS, W. (2004): "The role of attributions in customer satisfaction: a re-examination", Journal of Consumer Research, Vol. 31, No. 2, pp. 476-483.

VARGO, S.L.; NARAO, K.; HE, Y. Y MORGAN, F.W. (2007): "Satisfiers, dissatisfiers, criticals, and neutrals: A review of their relative effects on customer (dis)satisfaction", Academy of Marketing Science Review, Vol. 11, No. 2, pp. 1-19.

WEINER, B. (1985): "An attributional theory of achievement motivation and emotion", Psychological Review, Vol. 92, No. 4, pp. 548-573.

WEINER, B. (2000): "Attributional thoughts about consumer behavior", Journal of Consumer Research, Vol. 27, No. 3, pp. 382-387.

WESTBROOK, R.A. (1987): "Product consumption-based affective responses and postpurchase processes", Journal of Marketing Research, Vol. 24, pp. 258-270.

WESTBROOK, R.A. Y OLIVER, R.L. (1991): "The dimensionality of consumption emotion patterns and consumer satisfaction", Journal of Consumer Research, Vol. 18, pp. 84-91.

WIRTZ, J. Y BATESON, J.E.G. (1999): "Consumer satisfaction with services: integrating the environment perspective in services marketing into the traditional disconfirmation paradigm", Journal of Business Research, Vol. 44, pp. 55-66.

WIRTZ, J.; MATTILA, A.S. Y TAN, R.L.P. (2000): "The moderating role of target-arousal on the impact of affect on satisfaction-an examination in the context experiences", Journal of Retailing, Vol. 76, No. 3, pp. 347-365.

WOODRUFF, R.B.; SCHUMANN, D.W. Y GARDIAL, S.F. (1993): "Understanding value and satisfaction from the customer's point of view", Survey of Business, Vol. 28, pp. 33-40.

WOODRUFF, R.B. Y GARDIAL, S.F. (1996): Know Your customer: New Approaches to Understanding Customer Value and Satisfaction. Blackwell Publishers. 
YI, S. Y BAUMGARTNER, H. (2004): "Coping with negative emotions in purchase-related situations", Journal of Consumer Psychology, Vol. 14, No. 3, pp. 303.

YU, Y.T. Y DEAN, A. (2001): "The contribution of emotional satisfaction to consumer loyalty", International Journal of Service Industry Management, Vol. 12, No. 3, pp. 234-250.

ZEELENBERG, M. Y PIETERS, R. (2004): "Beyong valence in customer dissatisfaction: A review and new findings on behavioral responses to regret and disappointment in failed services", Journal of Business Research, Vol. 57, pp. 445-455. 